\title{
Editorial
}

\section{Preventing Foreign Bodies Injuries in Children: the Italy-Argentina Joint Research Programme 2011-2013}

\author{
Ambassador Guido La Tella*
}

Ambasciata d' Italia in Buenos Aires, Capital Federal, Argentina

I am very pleased to introduce this special issue of the Open Paediatric Medicine Journal, dedicated to the ItalyArgentina Joint Research Programme on Preventing Foreign Bodies Injuries in children.

Italy and Argentina have a long and successful tradition of scientific and technological cooperation, reflected in a wide range of agreements, scholarships and joint researches that take place every year.

The research on Preventing Foreign Bodies Injuries in children has been approved within the framework of the Executive Programme for 2011-2013 of the Agreement of Cultural, Scientific and Technological Cooperation between Italy and Argentina, signed in 1997 by the General Directorate for Cultural Cooperation of the Italian Ministry of Foreign Affairs (MAE) and the Ministry of Science, Teenology and Industrial Innovation of Argentina (Ministerio de Ciencia, Tecnología e Innovación Productiva - MINCYT).

The Italian Ministry of Foreign Affairs is financing the project for the whole period 2011-2013, given the high level of the institutions and researchers involved as well as the impact that it can have in the prevention and management of injuries by foreign body in the upper airways, which are one

Address correspondence to this author at the Ambasciata d' Italia in Buenos Aires, Calle Billinghurst 2577 (1425), Capital Federal, Argentina;

Tel: +54 114011 2100; Fax: +54 114011 2159;

E-mail: ambasciata.buenosaires@esteri.it of leading causes of death, by asphyxiation of the child, in ages $0-3$ years.

The goal of the project, led by Prof. Dario Gregori of the University of Padua and Dr. Hugo Rodriguez, of the Hospital de Pediatría Juan P. Garrahan in Buenos Aires, is indeed to create a network of collaboration within the institutions of the two countries to promote the development of a common protocol of prevention initiatives.

The exchange of ideas and researchers will help developing and laying the foundations for a lasting collaborative relationship and eventually lead to the implementation of a risk assessment procedure, in order to guide the processes of production and marketing of products sold in the global market.

This goal is fully consistent with the spirit of the Agreement between Italy and Argentina as well as the efforts of Global Public Health, which sees collaboration between countries as a preferential means for the prevention of injuries in the population.

The Embassy of Italy has been supporting the project since it was conceived and will sponsor in 2012 a seminar in Buenos Aires to present the results that the study has already achieved.

I congratulate again the Open Paediatric Medicine Journal for dedicating this issue to the cooperation between Italy and Argentina in such a delicate field as children's health and look forward to celebrating the completion of the project in 2013 . 